\title{
К вопросу выбора начальных аэродинамических условий в оценке тепловой безопасности объекта хранения тепловыделяющих материалов
}

\section{Амосов П.В.}

Институт проблем промышленной экологии Севера КНЦ PAH, Anamumbl, p.amosov@ksc.ru

Аннотация. Представлены результаты исследования по влиянию начальных аэродинамических условий на прогноз тепловой безопасности подземного объекта долговременного хранения отработавшего ядерного топлива. Доказано соблюдение требований тепловой безопасности функционирования объекта в консервативных условиях проветривания по величине расхода воздуха и значению коэффициента теплопроводности материала встроенной конструкции независимо от выбора начальных аэродинамических условий.

Ключевые слова: тепловыделяющие материалы, отработавшее ядерное топливо, численное моделирование, эффект плавучести, начальные условия, тепловая безопасность.

\section{On a question of choice of initial aerodynamic conditions on the assessment of thermal safety of underground storage facility of heat-producing materials}

\section{Amosov P.V.}

Institute of the Industrial Ecology Problems of the North KSC RAS, Apatity, p.amosov@ksc.ru

\begin{abstract}
The article presents the results of a study on the effect of initial aerodynamic conditions on the prediction of thermal safety of an underground facility for long-term storage of spent fuel. Compliance with the requirements of thermal safety of operation of the facility in conservative conditions of ventilation in air rate value and the value of the coefficient of thermal conductivity of the material of the built-in design regardless of the choice of initial aerodynamic conditions are proved.
\end{abstract}

Key words: heat-producing materials, spent fuel, numerical modeling, buoyancy effect, initial conditions, thermal safety.

\section{Введение}

Результаты исследований теплового режима подземного хранилища отработавшего ядерного топлива (ОЯТ) представлены в публикациях 2010 г. с использованием либо встроенной железобетонной конструкции (Амосов, Подшивалова, 2010а), либо железобетонных контейнеров (Амосов, Подшивалова, 2010б) и естественных (горная порода) защитных барьеров. В качестве модельного примера рассматривалась разработка специалистов Горного института КНЦ РАН по конструктивно-компоновочной схеме объекта (Мельников и др., 2003). В публикациях (Амосов, Подшивалова, 2010 a; 2010б) математическая модель основывалась на уравнениях неразрывности Навье-Стокса для вязкой несжимаемой жидкости и общем уравнении теплопереноса. В компьютерной модели отсутствовал важный член уравнения Навье-Стокса, который отвечает за эффект плавучести, что снизило ценность выполненных исследований. Как отметили авторы работы (Амосов, Подшивалова, 2010 б), их попытки включить в уравнения Навье-Стокса силы плавучести в приближении Буссинеска оказались неудачными.

Исправляя указанный момент с учетом накопленного за прошедшие годы опыта численного моделирования аэротермодинамических процессов с учетом сил плавучести, в статье изложены результаты исследования по влиянию выбора начальных аэродинамических условий на аэротермодинамику атмосферы подземного модуля хранения ОЯТ (встроенная конструкция) и результаты прогнозных оценок тепловой безопасности объекта.

Рассмотрено два варианта начальных аэродинамических условий:

- вариант 1 - решение стартует практически с нулевых значений компонентов скорости;

- $\quad$ вариант 2 - решению предшествует этап расчета стационарного поля скорости, который выполняется в приближении несжимаемой жидкости. 


\section{Параметры модели}

Геометрические параметры модуля встроенной железобетонной конструкции приведены в работе Амосова П.В. и Подшиваловой А.В. (2010а). Там же указаны используемые начальные и граничные условия, значения теплофизических параметров, динамика мощности остаточных тепловыделений. Режим неизотермического потока моделируется с помощью уравнений неразрывности Навье-Стокса, описывающих связь скорости движения воздуха и давления с учетом температурного фактора, классического уравнения переноса тепла (Егоров, 2006). Используется приближение «несжимаемого идеального газа», т.е. свойства воздуха зависят только от температуры.

С учетом ранее сделанного вывода о безусловном обеспечении тепловой безопасности при высоких значениях эффективного коэффициента теплопроводности области тепловыделений $k_{w}$ (Амосов, Подшивалова, 2010а) значения этого параметра в численных экспериментах принимали значения 1.0 и $2.0 \mathrm{Bт} /($ мК). Для расхода воздуха $Q$ по-прежнему использовались значения $0.06,0.18$ и $0.30 \mathrm{M}^{3} / \mathrm{c}$.

\section{Аэродинамические характеристики потоков}

Принципиальное отличие результатов численных экспериментов 2010 г. (Амосов, Подшивалова, 2010 а) и представляемых в статье заключается в структуре воздушных потоков в объекте хранения. Если в модели несжимаемой жидкости структура воздушных потоков при рассмотренных расходах воздуха идентична на всем протяжении решения нестационарной задачи теплопереноса (отличие только в значениях компонентов скорости для разных расходов), то учет влияния тепла на аэродинамику делает проблему по-настоящему нестационарной. В качестве примера на рисунке (a, б) приведены «мгновенные» картинки скоростных полей на момент времени моделирования 5 лет для $k_{w}$ равном $1.0 \mathrm{BT} /($ м К) при вариации $Q$ (верхний 0.06 , средний 0.18 и нижний $0.30 \mathrm{~m} / \mathrm{c}$ ). Рис. а отвечает варианту 1 и рис. б варианту 2 , соответственно (использована опция «нормализованных» стрелок).

Визуальное сравнение «мгновенных» картинок позволяет качественно оценить влияние величины расхода поступающего воздуха. Например, на рис. (a, б) при расходе $0.06 \mathrm{~m}^{3} / \mathrm{c}$ (верхние картинки) обратный поток воздуха в середине объекта занимает практически половину высоты пространства между поверхностью железобетонной конструкции и кровлей. С ростом расхода воздуха до $0.18 \mathrm{~m}^{3} / \mathrm{c}$ (средние картинки) область обратного потока вдоль кровли сокращается примерно на треть. А при дальнейшем росте расхода до $0.30 \mathrm{~m}^{3} / \mathrm{c}$ (нижние картинки) обратный поток на рассматриваемый момент времени практически исчезает на рис. а и размещается лишь вдоль кровли на рис. б. Другими словами, в ситуации максимального расхода воздуха с течением времени с учетом монотонного спада объемной мощности остаточных тепловыделений структура потока будет приближаться к структуре поля скорости для модели несжимаемой жидкости.

Эффект влияния начальных аэродинамических условий на структуру поля скорости удобно проследить на следующих примерах. При минимальном расходе воздуха 0.06 м³/с (верхние картинки) различия на рис. а и б можно обнаружить лишь при детальном рассмотрении. Для расхода воздуха $0.18 \mathrm{~m}^{3} / \mathrm{c}$ (средние картинки) на рис. а и б отчетливо видно различие в структуре потока в левой верхней области объекта (до конструкции с ОЯТ), а также под кровлей объекта хранения по краям встроенной конструкции. При максимальном расходе воздуха $0.30 \mathrm{~m}^{3} / \mathrm{c}$ (нижние картинки) заметные различия в структуре поля скорости рис. а и б хорошо видны в верхней части под кровлей объекта (обратный поток в варианте 2, чего нет в варианте 1) и справа ближе к выходу истечения воздуха.

Динамика формирования скоростного поля проанализирована на примере горизонтальной компоненты вектора скорости в трех сечениях модели (отметки 70, 80 и 90 м вдоль горизонтальной оси) для всех параметров варьирования и двух вариантов задания начальных аэродинамических условий. Можно отметить несколько моментов. В первую очередь это существенное различие в конвективном механизме переноса тепла, который является доминирующим в воздушной среде. Если в модели несжимаемой жидкости (Амосов, Подшивалова, 2010а) имеем практически однонаправленный перенос тепла к выходу из объекта хранения, то в модели учета плавучести конвективный перенос тепла варьируется как во времени, так и в пространстве (независимо от выбора варианта на- 

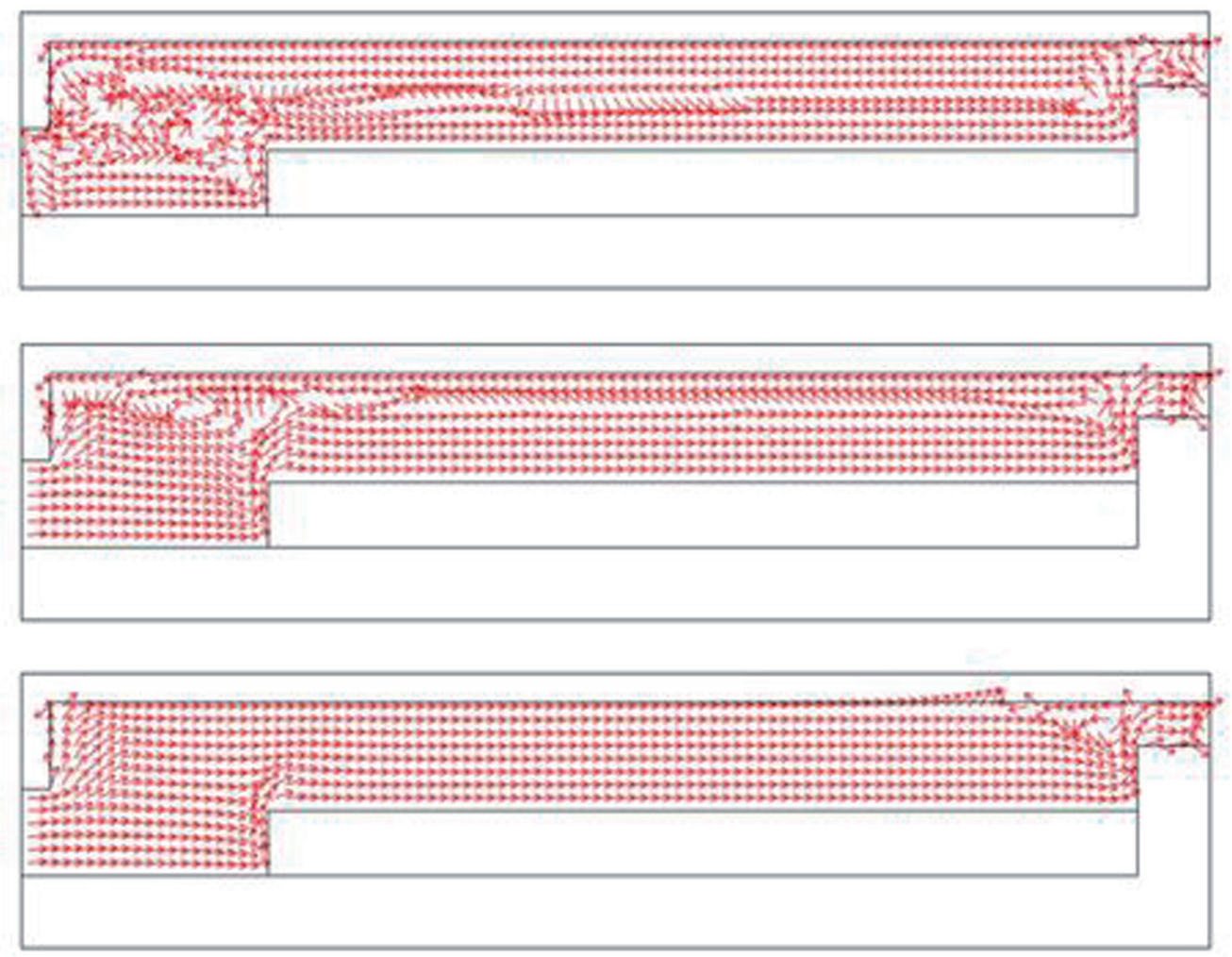

a
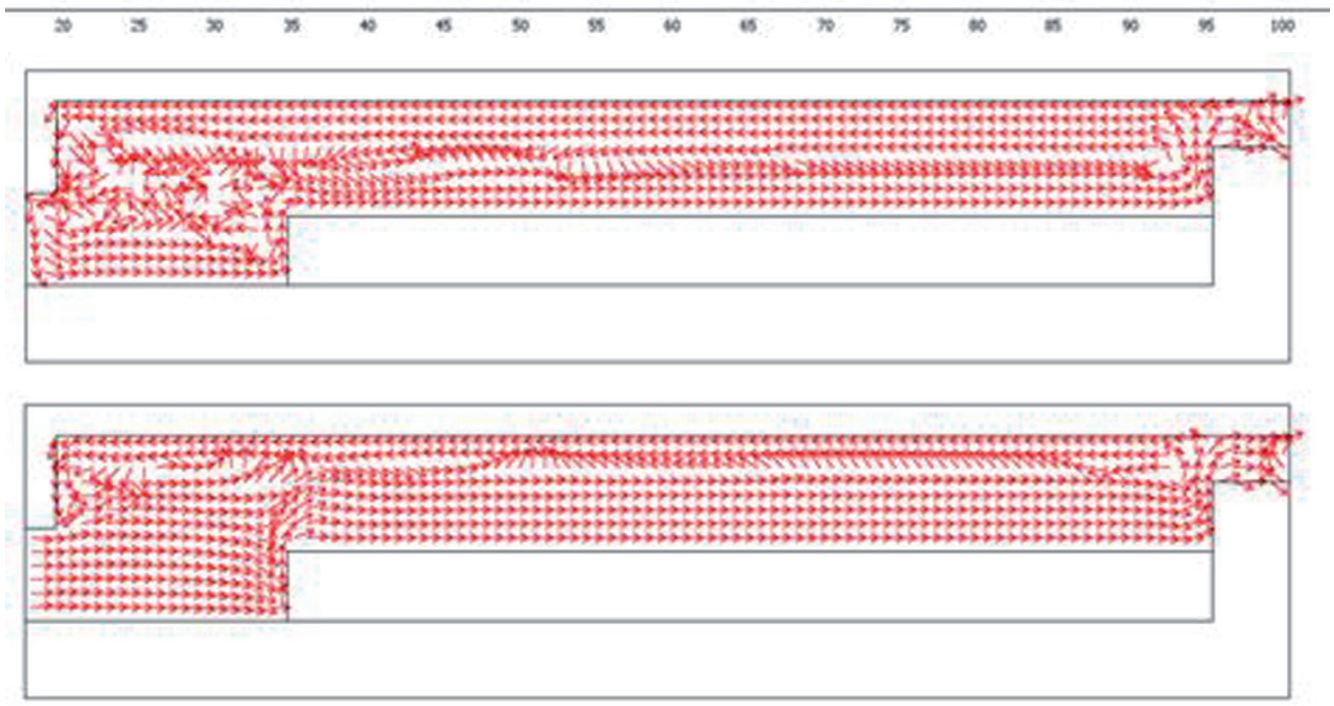

б

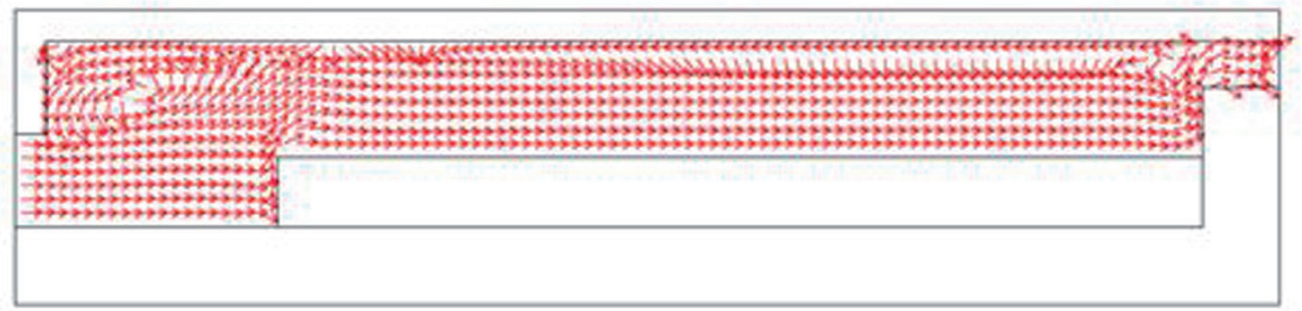

Рис. «Мгновенная» картина структуры поля скорости при $k_{w}$ равном $1.0 \mathrm{BT} /($ м.К) при вариации $Q$ (верхний 0.06 , средний 0.18 и нижний $\left.0.30 \mathrm{~m}^{3} / \mathrm{c}\right)$ : а) вариант 1 и б) вариант 2.

Fig. "Instantaneous" picture of velocity field structure at $k_{w}$ equal to $1.0 \mathrm{~W} /(\mathrm{m} \cdot \mathrm{K}$ ) with variation $Q$ (up 0.06 , middle 0.18 and down $0.30 \mathrm{~m}^{3} / \mathrm{sec}$ ): a) variant 1 and б) variant 2 . 
чальных аэродинамических условий). Во-вторых, выбор начальных аэродинамических условий вносит свой вклад, хотя и менее значительный в конечный результат теплового состояния материалов.

\section{Тепловая безопасность объекта хранения}

Для ответа на вопрос об условиях выполнения критериальных значений температуры проанализированы пространственно-временные распределения температуры для указанных выше параметров варьирования. В первую очередь интерес представляют критериальные значения температуры (Сорокин и др., 1989): в области скального массива (не выше $\left.100^{\circ} \mathrm{C}\right)$ и на поверхности железобетонной конструкции (не выше $85^{\circ} \mathrm{C}$ ).

Анализ распределений температуры выполнен в указанных выше сечениях модели (отметки 70, 80 и 90 м) для всех параметров варьирования.

Самый важный момент - тепловая безопасность объекта хранения неперерабатываемых типов ОЯТ подтверждается. Однако, если в модели несжимаемой жидкости прогрев скального массива прогнозировался до уровня $40^{\circ} \mathrm{C}$, а поверхности инженерной конструкции до $50^{\circ} \mathrm{C}$ (Амосов, Подшивалова, 2010 a), то в модели, учитывающей эффект плавучести (независимо от выбора начальных аэродинамических условий), прогнозные значения температуры иные.

Отметим несколько общих закономерностей поведения проанализированных графических температурных зависимостей:

- $\quad$ координаты точек с максимальными значениями температуры изменяются не только во времени, что естественно для нестационарной задачи, но и при вариации значений $k_{w}$ и $Q$;

- уменьшение $Q$ приводит к прогревам (в разной степени) всех без исключения областей модели;

- увеличение $k_{w}$ снижает прогнозные значения температуры скального массива вблизи подошвы и области тепловыделений, но увеличивает температуру поверхности инженерной конструкции, воздуха исходящей струи и скального массива кровли.

В таблице представлены максимальные температуры в различных областях модели при вариации $k_{w}$ и $Q$ для обоих вариантов начальных аэродинамических условий.

Таблица. Значения максимальных температур в различных областях модели при вариации $k_{w}$ и $Q$ (числитель - вариант 1 , знаменатель - вариант 2 ), ${ }^{\circ} \mathrm{C}$.

Table. Values of maximum temperatures in different regions of the model with variation $k_{w}$ and $Q$ (numerator - variant 1 , denominator - variant 2 ), ${ }^{\circ} \mathrm{C}$.

\begin{tabular}{|c|c|c|c|c|c|c|}
\hline \multirow{2}{*}{$k_{w}, \mathrm{~B} \mathrm{~s} /(\mathrm{м} \cdot \mathrm{K})$} & \multirow{2}{*}{$Q, \mathrm{~m}^{3} / \mathrm{c}$} & \multicolumn{5}{|c|}{ Контролируемые области модели } \\
\cline { 3 - 7 } & & Тепловыделения & Поверхность & Гранит_1 & Гранит_2 & Воздух \\
\hline \multirow{3}{*}{1.0} & 0.06 & $90.2 / 90.3$ & $32.4 / 32.6$ & $90.1 / 90.2$ & $33.4 / 33.7$ & $33.4 / 33.7$ \\
\cline { 2 - 7 } & 0.18 & $82.7 / 83.5$ & $22.5 / 25.0$ & $82.6 / 83.4$ & $22.3 / 24.4$ & $22.3 / 24.4$ \\
\cline { 2 - 7 } & 0.30 & $82.3 / 83.4$ & $21.8 / 24.9$ & $82.2 / 83.3$ & $19.0 / 19.5$ & $19.0 / 19.5$ \\
\hline \multirow{3}{*}{2.0} & 0.06 & $64.2 / 64.7$ & $33.5 / 33.7$ & $64.2 / 64.6$ & $35.2 / 35.1$ & $35.2 / 35.1$ \\
\cline { 2 - 7 } & 0.18 & $56.4 / 56.4$ & $24.9 / 25.2$ & $56.4 / 56.4$ & $22.8 / 22.8$ & $22.8 / 22.8$ \\
\cline { 2 - 7 } & 0.30 & $55.0 / 55.0$ & $22.3 / 22.4$ & $55.0 / 55.0$ & $19.3 / 19.2$ & $19.3 / 19.2$ \\
\hline
\end{tabular}

Примечание. Гранит_1 - подошва и Гранит_2 - кровля выработки.

Максимальный разогрев имеет место при минимальных значениях расхода воздуха и эффективного коэффициента теплопроводности области тепловыделений. На расчетное время 5 лет прогнозное значение температуры скального массива достигает $90^{\circ} \mathrm{C}$, что близко к критериальному. Но следует обратить внимание, что к этому моменту времени скорость разогрева заметно снижается. А с учетом спада мощности остаточных тепловыделений можно гарантированно утверждать, что превышения порогового значения не произойдет. Кроме того, в реальных условиях отток тепла 
будет происходить в больших объемах скальной породы, что дополнительно снизит значение прогнозируемой температуры.

Анализ распределений температуры и расчетных данных таблицы свидетельствует, что эффект выбора начальных аэродинамических условий присутствует. Например, все графики температуры варианта 2 располагаются немного выше соответствующих графиков варианта 1 . Количественные показатели эффекта иллюстрируются данными таблицы. При $k_{w}$ равном $1.0 \mathrm{BT} /(\mathrm{м} \cdot \mathrm{K})$ выбор начальных аэродинамических условий в наименьшей степени отражается на максимальных температурах для области тепловыделений и скального массива вблизи подошвы. Для варианта 2 прогнозируемый рост температуры в сравнении с вариантом 1 составляет не более $1 \%$. Тогда как увеличение максимальной температуры поверхности конструкции для варианта 2 по сравнению с вариантом 1 достигает почти $14 \%$. При $k_{w}$ равном $2.0 \mathrm{BT} /($ м.К) эффект начальных аэродинамических условий практически исчезает.

\section{Заключение}

На основании проведённого исследования можно сформулировать следующие выводы:

- построены компьютерные модели аэротермодинамики подземного объекта долговременного хранения ОЯТ в варианте встроенной железобетонной конструкции в двухмерной постановке для двух вариантов начальных аэродинамических условий. Учет эффекта плавучести реализован в приближении «несжимаемого идеального газа»;

- рассмотрено два варианта начальных аэродинамических условий: в варианте 1 совместное решение системы уравнений начинается практически с нулевых значений компонентов скорости, в варианте 2 есть предварительный этап расчета стационарного поля скорости, который выполняется в приближении несжимаемой жидкости.

- выполнен сравнительный анализ расчетных аэротермодинамических параметров в приближении «несжимаемого идеального газа» с результатами численных экспериментов в модели несжимаемой жидкости для обоих вариантов начальных аэродинамических условий;

- продемонстрированы принципиальные отличия в структурах скоростных полей, прогнозируемых в объекте хранения на базе двух моделей несжимаемой жидкости и «несжимаемого идеального газа» и для двух вариантов начальных аэродинамических условий, при вариации расхода поступающего воздуха и эффективного коэффициента теплопроводности встроенной конструкции;

- доказана тепловая безопасность функционирования объекта для режима вынужденной конвекции воздуха для обоих вариантов начальных аэродинамических условий: разогревы поверхности встроенной конструкции и вмещающего скального массива не превышают установленных критериальных значений;

- выполнен анализ максимальных значений температуры в различных областях моделей для обоих вариантов начальных аэродинамических условий при вариации параметров моделей.

\section{Литература}

1. Амосов П.В., Подшивалова А.В. Моделирование теплового режима подземного объекта хранения тепловыделяющих радиационно опасных материалов // Вестник МГТУ. 2010 а. Т. 13. № 3. С. 562-566.

2. Амосов П.В., Подшивалова А.В. Особенности теплового режима подземного объекта изоляции тепловыделяющих материалов (контейнерный вариант) // Известия вузов Ядерная энергетика. 2010 б. № 3. С. $133-140$.

3. Егоров В.И. Применение ЭВМ для решения задач теплопроводности. Учебное пособие. СПб.: СПб ГУ ИТМО, 2006. $77 \mathrm{c}$.

4. Мельников Н.Н., Конухин В.П., Наумов В.А., Амосов П.В., Гусак С.А., Наумов А.В., Катков Ю.Р. Отработавшее ядерное топливо судовых энергетических установок на европейском Севере России. Ч.ІІ. Апатиты: КНЦ РАН, 2003. 209 с.

5. Сорокин В.Т., Козлов А.Е., Пучкова О.К. Теплотехнические аспекты захоронения отвержденных радиоактивных отходов в геологических формациях: препринт: препринт инв. № 89-05353. Л.: ВНИПИЭТ, 1989. $42 \mathrm{c}$. 